\title{
Miniature Fiber-Optic Magnetic Field Sensor Based on Ampere Force and Fiber Laser
}

\author{
Weiren CHENG, Tianming LUO, Linghao CHENG*, Hao LIANG, and Bai-ou GUAN \\ Institute of Photonics Technology, Jinan University, Guangzhou 510632, China \\ *Corresponding author: Linghao CHENG？ E-mail: chenglh@ieee.org
}

\begin{abstract}
A compact fiber-optic magnetic field sensor is proposed by packaging an orthogonal dual-frequency fiber grating laser and a copper wire with alternating electrical current together inside epoxy resin. The alternating current generates Ampere force in a magnetic field, which changes the birefringence of the fiber laser and hence tunes the frequency of the beat signal after photodetection. The magnetic flux density can then be detected by measuring the frequency change of the beat signal. The sensitivity of the sensor can be tuned with a maximum response of $35.21 \mathrm{kHz} / \mathrm{kGs}$ demonstrated. Moreover, the sensor shows good immunity to environment interference.
\end{abstract}

Keywords: Fiber laser; optical magnetic field sensors; fiber-optic sensors; Ampere force

Citation: Weiren CHENG, Tianming LUO, Linghao CHENG, Hao LIANG, and Bai-ou GUAN, "Miniature Fiber-Optic Magnetic Field Sensor Based on Ampere Force and Fiber Laser," Photonic Sensors, 2020, 10(4): 291-297.

\section{Introduction}

Magnetic field is of great importance in various areas and its detection has always been a research hotspot in the world [1], attracting the attention of institutions in many countries. Compared with traditional sensing methods, fiber-optic based magnetic field sensing technology shows advantages in small size, large bandwidth, light weight, and immunity to electromagnetic interference [2]. Various technologies have been proposed to implement fiber-optic magnetic field sensors, such as magnetic force, Lorentz force, Faraday effect, magnetostrictive effect, and magnetic fluid materials [3-6]. Among them, Faraday effect is popular because it can directly measure magnetic field. However, since the Verdet constant of silica fiber is small, the sensitivity of Faraday effect based methods is normally quite low $[7,8]$ even with magneto-optical crystal introduced to increase the sensitivity [9]. Sensing techniques based on other mechanisms typically measure magnetic field indirectly through external transducers to increase sensitivity, such as through magnetostrictive transducer and Ampere force [10-12]. Such schemes generally convert magnetic field to an intermediate physical measurand which can be measured through fiber-optics with high sensitivity. Recently, sensing techniques using orthogonal dual-frequency fiber grating lasers have attracted widespread attentions. The dual-polarization mode in the same longitudinal mode of an orthogonal dual frequency laser produces a beat signal after photodetection, and its frequency varies with changes in birefringence within the laser cavity [13]. A very low cavity birefringence change of $10^{-8}$ can cause the beat frequency to vary in megahertz range. Therefore, the orthogonal dual-frequency fiber grating laser can reach quite high sensitivity. Moreover, demodulation of the beat signal is in an electronic domain and the

Received: 12 March 2020 / Revised: 12 May 2020

(C) The Author(s) 2020. This article is published with open access at Springerlink.com

DOI: $10.1007 / \mathrm{s} 13320-020-0590-5$

Article type: Regular 
cost is fairly lower than that of processing in optical domain [14]. Based on the orthogonal dual-frequency fiber grating laser, some fiber-optic magnetic field sensors have been proposed, such as those employing magnetostrictive materials [15], magnet force, and Ampere force [16, 17].

Besides sensitivity, suppression of interference is also of great importance to a magnetic field sensor, especially for those working for measurement of stationary and low-frequency magnetic field. For fiber-optic magnetic field sensors, such interference can come from temperature changes, vibration, and other environmental disturbances [18]. These disturbances mainly manifest themselves in lowfrequency band [19]. Therefore, sensors measuring magnetic field directly in low-frequency band are easily interfered by environmental disturbances. To combat this, we have proposed a scheme based on Ampere force, which can measure a stationary magnetic field in high-frequency band of kilohertz. By working in high-frequency band, environmental disturbances of low frequency can be resisted and measurement is therefore more reliable. However, the scheme is not in a compact structure and hence cannot work as a full functional sensor with high reliability.

In this paper, based on an orthogonal dual-frequency fiber grating laser and alternating Ampere force, we realize a miniature fiber-optic magnetic field sensor. The orthogonal dualfrequency fiber grating laser is attached to a copper wire and both of them are packaged by epoxy resin to form a reliable and functional magnetic field sensor in a compact size. The sensitivity of the sensor can be optimized at the resonance peak of some frequency of alternating Ampere force, which also maintains sensor's stability in disturbances.

\section{Principles and experiments}

\subsection{Principles}

Figure 1(a) shows the structure of the proposed sensor. The fiber laser and the copper wire are located at the center of the sensor and surrounded by polymer. Figure 1(b) shows the working principle of the sensor. When the copper wire is driven by an alternating electrical current source, in a magnetic field perpendicular to the current, the copper wire will exert a force onto the fiber laser due to the Ampere force generated by the current. This force will change the birefringence of the laser cavity and hence change the beat frequency of the laser output according to the following equation provided the two polarizations are of the same longitudinal mode [20]:

$$
\Delta v=\frac{c}{n_{0} \lambda_{0}} B
$$

where $\Delta v$ is the beat frequency, $B$ is the birefringence denoted by the refractive index difference between the two polarization modes inside the laser cavity, $c$ is the light speed in vacuum, $n_{0}$ is the average refractive index of the fiber, and $\lambda_{0}$ is the laser wavelength. The beat frequency is therefore directly related to the birefringence linearly. For a practical magnetic field sensing, the birefringence can come from 3 sources. The first one is the intrinsic fiber birefringence $\mathbf{B}_{I}$. The second is due to environmental disturbance and noted by $\mathbf{B}_{D}$. The third comes from Ampere force and given by $\mathbf{B}_{A}$. When $\mathbf{B}_{D}$ and $\mathbf{B}_{I}$ are much smaller than $\mathbf{B}_{A}$, the total birefringence can be expressed as the vector summation of the three and given by

$$
\mathbf{B}=\mathbf{B}_{I}+\mathbf{B}_{D}+\mathbf{B}_{A} .
$$

Because $\mathbf{B}_{I}$ is an invariable vector and $\mathbf{B}_{D}$ is generally in a low-frequency band, if $\mathbf{B}_{A}$ is in a high-frequency band, the birefringence due to magnetic field through Ampere force can be detected without disturbance. It is well known that Ampere force generated by an electrical current in a perpendicular magnetic field is given by

$$
\mathbf{F}_{A}=L \mathbf{I} \times \mathbf{H}
$$

where $\mathbf{H}$ is the magnitude of magnetic field, $\mathbf{I}$ is the electrical current, and $L$ is the length of electrical 


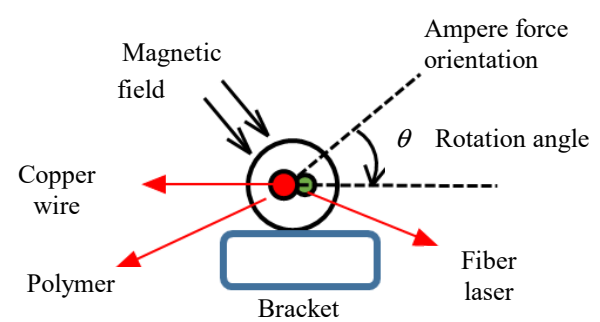

(a)

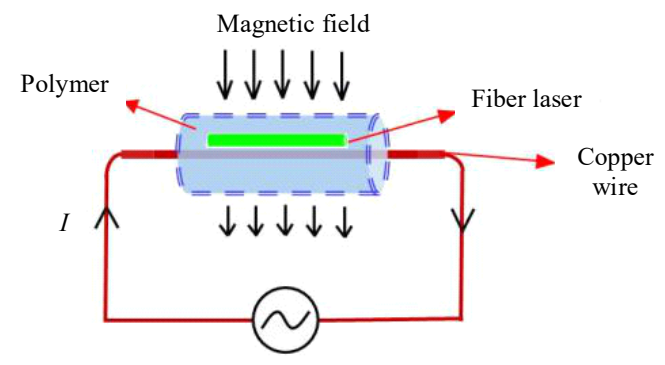

(b)

Fig. 1 Structure and principle of the sensor: (a) the cross-section of the fiber-optic magnetic field sensor and (b) working principle of the fiber-optic magnetic field sensor.

current experiencing the magnetic field. It then shows that if $\mathbf{I}$ is an alternating current, the Ampere force $\mathbf{F}_{A}$ and hence the birefringence $\mathbf{B}_{A}$ are also alternating at the same frequency value. The magnetic field can then be measured by detecting at the driving frequency of the current and the environmental disturbance can be largely suppressed. If the driving frequency is $\omega$, the beat frequency of the laser output can be expressed by [21]

$$
\begin{aligned}
\Delta v= & \frac{c}{n_{0} \lambda_{0}}\left|\mathbf{B}_{I}+\mathbf{B}_{D}\right|+ \\
& \frac{2 c n_{0}^{2}\left(p_{11}-p_{12}\right)\left(1+v_{p}\right) \cos (2 \theta)}{\pi r \lambda_{0} E} . \\
& \eta|\mathbf{I} \times \mathbf{H}| \cos (\omega t)
\end{aligned}
$$

where $p_{11}$ and $p_{12}$ are the elements of the strain-optical tensor of the fiber material, $n_{0}$ is the average refractive index of the fiber, $\lambda_{0}$ is the laser wavelength, $v_{p}$ is Poisson's ratio, $r$ is the fiber radius, $t$ is time, $\theta$ is the angle of the applied force with respect to the fast axis, and $E$ is the Young's modulus of the silica fiber. Because the length of the fiber laser is much shorter than that of the copper wire in the sensor, only part of the Ampere force is exerted to the fiber laser. Therefore, a coefficient $\eta$ is introduced in (4) to account for this. Equation(4) shows that the magnetic field induced beat frequency change is located at a frequency of $\omega$ and is well separated from low-frequency disturbances.

\subsection{Fabrication of the fiber-optic magnetic field sensor}

The fiber laser is a dual-polarization distributed Bragg reflector (DBR) fiber laser engraved on the $\mathrm{Er} / \mathrm{Yb}$ co-doped fiber (EY305) with two grating lengths of $4 \mathrm{~mm}$ and $3.5 \mathrm{~mm}$, and a grating spacing of $5 \mathrm{~mm}$. The laser is pumped at $975 \mathrm{~nm}$ with an absorption coefficient at $975 \mathrm{~nm}$ of $1250 \mathrm{~dB} / \mathrm{m}$. The wavelength of the laser output is $1550.6 \mathrm{~nm}$, with intensity of $-25 \mathrm{dBm}$, and the beat frequency of its two polarizations is $1.348 \mathrm{GHz}$. The diameter of the copper wire is $1 \mathrm{~mm}$. The fiber laser is then tightly attached to the copper wire and both of them are immersed in liquid epoxy resin hold in a transparent plastic round mold with a diameter of $6 \mathrm{~mm}$. It takes 12 hours for the epoxy resin to cure automatically in the mold and then the proposed miniature fiber-optic magnetic field sensor is ready for measurement. The entire sensor has a diameter of $6 \mathrm{~mm}$ and a length of $5 \mathrm{~cm}$ packaged by solid epoxy resin, making the inner fiber grating laser well isolated from outside environment disturbances. Because the DBR fiber laser is only $12.5 \mathrm{~mm}$ in length, the working length of the copper wire inside the package is also only $12.5 \mathrm{~mm}$.

\subsection{Experimental setup}

The experimental setup is shown in Fig. 2. The fiber laser is attached to a copper wire and both of them are surrounded by polymer of epoxy resin to form a fiber-optic magnetic sensor. The sensor is installed on a rotating bracket to enable orientation measurement. The fiber laser is pumped by a pump laser of $979 \mathrm{~nm}$ through a wavelength division multiplexer (WDM). The output of the fiber laser is directed to a photodetector through the WDM and an isolator. To make the two orthogonal polarization modes beat with each other, a polarization controller (PC) followed by a polarizer is inserted before the 
photodetector. The output of the photodetector is then analyzed by a radio frequency (RF) spectrum analyzer. The copper wire inside the sensor is connected to an electrical current source. An alternating current (AC) flows through the copper wire. The sensor is placed between two electrical magnets to measure the magnetic field.

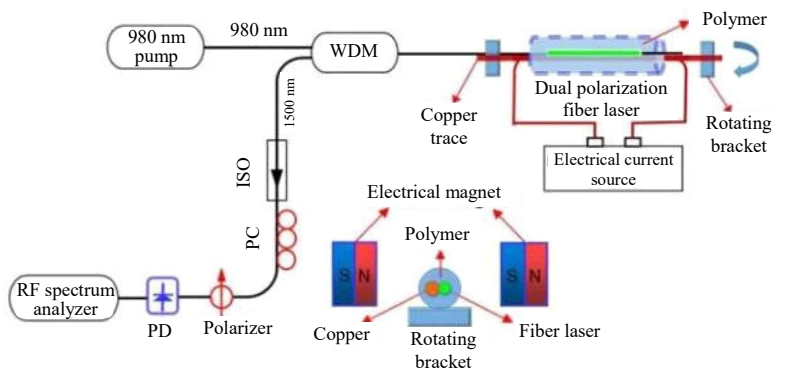

Fig. 2 Experimental setup for the proposed fiber-optic magnetic field sensor. (ISO: isolator; PC: polarization controller; PD: photodetector.)

\section{Results and discussion}

\subsection{Frequency response of the sensor}

When the copper wire is carrying $\mathrm{AC}$ current, it is actually slightly vibrating in the magnetic field due to the Ampere force. Because the copper wire is bounded by the solid polymer, the acoustic impedance difference between copper and polymer, and air makes the response of the sensor to such vibration frequency dependent and some resonance appear in frequency domain. Therefore, the frequency response of the sensor is measured. The electrical response of the copper wire to $\mathrm{AC}$ current is measured at first by sampling the current through a resistor and it shows the response is very flat at a frequency below $100 \mathrm{kHz}$. It then ensures that the measured response of the sensor is solely due to vibration by injecting current to the copper wire at frequency below $100 \mathrm{kHz}$. By tuning the frequency of the $\mathrm{AC}$ current, the peak-to-peak beat frequency variation is recorded as the response of the sensor to the Ampere force at the frequency. The finally obtained response of the sensor is plotted in Fig. 3, which is measured in a magnetic field of $3 \mathrm{kGs}$ and at a constant peak-to-peak AC current level of $1 \mathrm{~A}$. The results in Fig. 3 show two resonance peaks with the central frequency of the lower one at around $12 \mathrm{kHz}$.

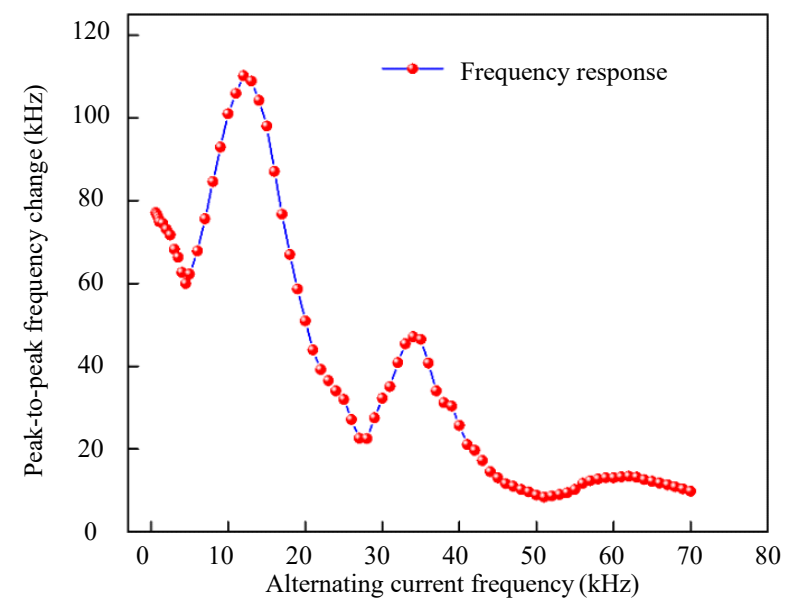

Fig. 3 Measured response of the sensor to different AC frequencies in a magnetic field of $3 \mathrm{kGs}$ and at a constant peak-to-peak AC current level of $1 \mathrm{~A}$.

\subsection{Beat frequency variation amplitude for various alternative current amplitudes}

The frequency of the AC current is then fixed at $12 \mathrm{kHz}$ to maximize the sensor response and the current level is tuned to measure the response of the sensor in a magnetic field of $3 \mathrm{kGs}$. The results are shown in Fig. 4. As expected by (4), the linearity of the sensor response is quite good. The slope of the linear fitting of the measured data is $105.728 \mathrm{kHz} / \mathrm{A}$. Larger current can increase the responsivity of the sensor and hence the sensitivity of the sensor can be electronically tuned through tuning the $\mathrm{AC}$ current level.

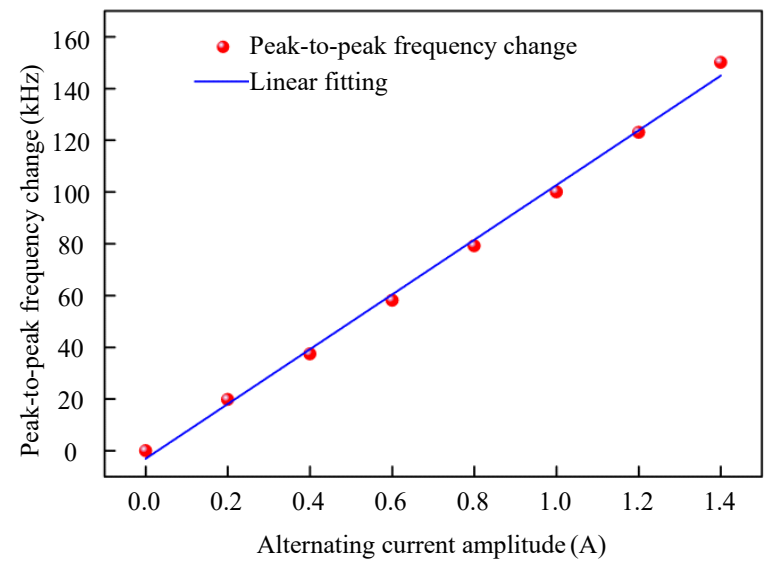

Fig. 4 Beat frequency variation amplitudes for various alternative current amplitudes. The current is alternating at $12 \mathrm{kHz}$ and the magnetic flux density is $3 \mathrm{kGs}$. 


\subsection{Beat frequency variation amplitude for various magnetic flux density}

Figure 5 shows the beat frequency variation amplitude for various magnetic flux density. The results are obtained at an $\mathrm{AC}$ current of peak-to-peak level of $1 \mathrm{~A}$ and alternating at $12 \mathrm{kHz}$. The results also confirm that the amplitude of the beat frequency is linearly related to the magnetic field amplitude as expected by (4). The slope of the linear fitting of the measured data is $35.21 \mathrm{kHz} / \mathrm{kGs}$ for a positive magnetic field and is $33.845 \mathrm{kHz} / \mathrm{kGs}$ for a negative magnetic field. The slight difference of the two slopes may be due to measurement errors.

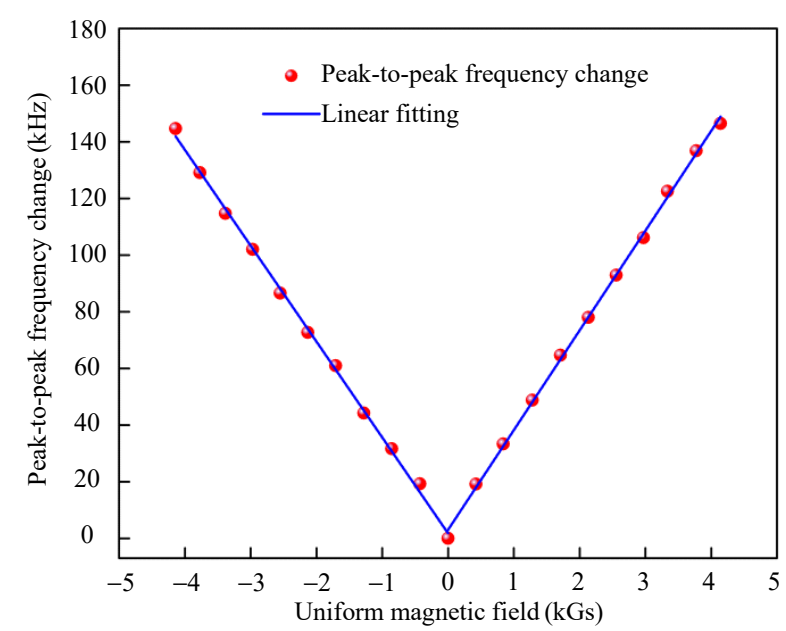

Fig. 5 Beat frequency variation amplitudes for various magnetic flux density. The current is alternating at $12 \mathrm{kHz}$ with peak-to-peak amplitude of $1 \mathrm{~A}$.

\subsection{Beat frequency variation amplitude for various rotation angles of the sensor}

Because the fiber laser is a dual-polarization fiber laser, it is actually directional and sensitive to the orientation of the Ampere force. If the Ampere force is aligned to one axis of the birefringence of the fiber laser cavity, the response of the sensor will be maximized or minimized.

The directivity of the sensor is measured and shown in Fig. 6. The sensor is mounted on a rotatable bracket and its responses at various orientation angles in a magnetic field are recorded as shown in Fig. 1(a). The current is alternating at $12 \mathrm{kHz}$ with peak-to-peak amplitude of $1 \mathrm{~A}$ and the magnetic flux density is $3 \mathrm{kGs}$. The directivity is clearly identified with the maximum response of about $120 \mathrm{kHz}$ at an angle of $90^{\circ}$ and the minimum response of about $30 \mathrm{kHz}$ at an angle of $0^{\circ}$. The directivity is then about $12 \mathrm{~dB}$. Therefore, the proposed sensor may be useful in measuring the vector of a magnetic field.

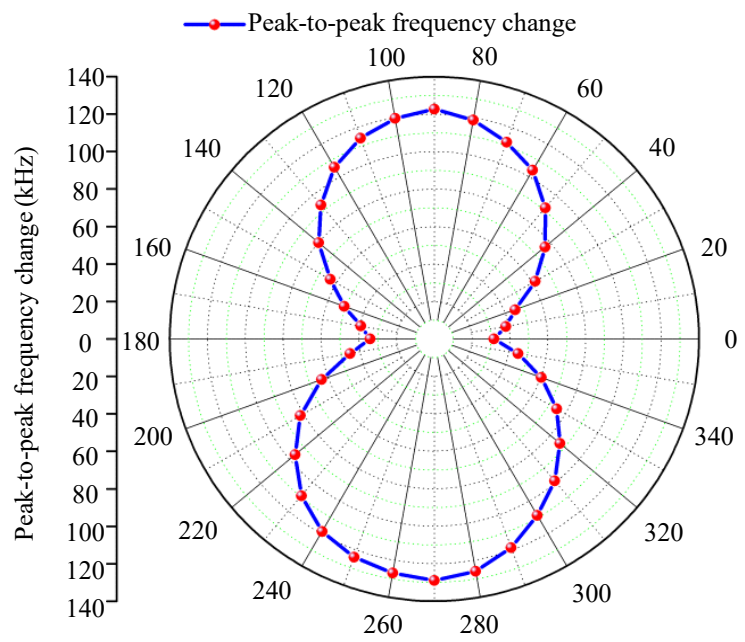

Fig. 6 Beat frequency variation amplitudes for various rotation angles of the sensor. The current is alternating at $12 \mathrm{kHz}$ with peak-to-peak amplitude of $1 \mathrm{~A}$ and the magnetic flux density is $3 \mathrm{kGs}$.

\subsection{Comparison of the spectra with and without signal due to Ampere force}

The signal-to-noise ratio (SNR) performance of the magnetic field sensor at the resonance frequency of $12 \mathrm{kHz}$ is investigated and shown in Fig. 7. The peak-to-peak amplitude of AC current is $1 \mathrm{~A}$ and the magnetic flux density is set to $6.35 \mathrm{kGs}$. The solid and the short dot curves show the spectra with and without AC current applied, respectively. The spectrum without AC current applied gives the noise level of the system. The comparison of the two spectra gives the SNR and it is read as about $54 \mathrm{~dB}$ at the resonance frequency of $12 \mathrm{kHz}$. Moreover, the noise level is much higher at the resonance frequency below $5 \mathrm{kHz}$. The information of the magnetic field is shifted to $12 \mathrm{kHz}$ by the alternating Ampere force, which makes the measurement less disturbed by environmental interference. Note that the power values shown in Fig. 7 are only in sense of 
relative magnitude because they are calculated after analog-to-digital conversion and then spectral analysis in digital domain.

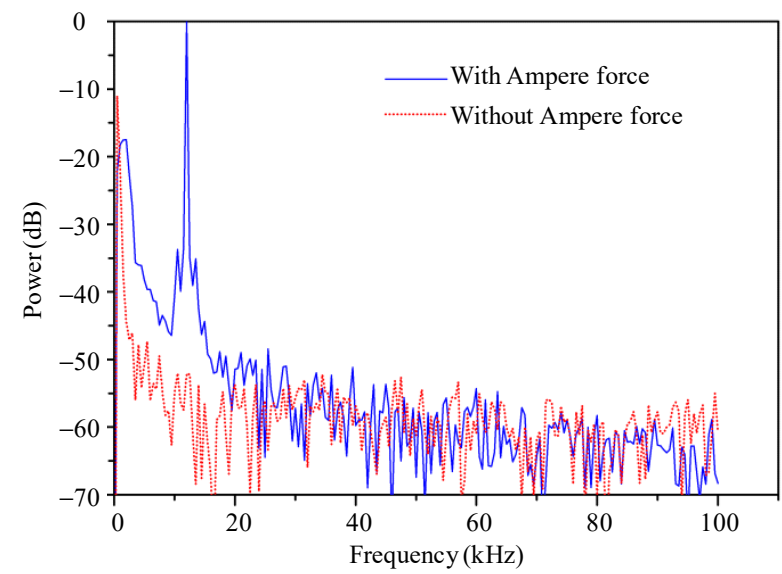

Fig. 7 Comparison of the spectrum with and without signal due to Ampere force.

\section{Conclusions}

In conclusion, a dual-polarization fiber laser and Ampere force based fiber-optic magnetic field sensor are proposed and realized in a compact size by incorporating a fiber laser and a copper wire carrying AC current into a miniature solid polymer package of epoxy resin. The package size is about $6 \mathrm{~mm}$ in diameter and $5 \mathrm{~cm}$ in length. The sensor shows good linearity and is capable of directional measurement. Its performance can be maximized at its resonance peak by setting the alternating frequency of the AC current to $12 \mathrm{kHz}$. An SNR of about $54 \mathrm{~dB}$ is achieved with the peak-to-peak amplitude of $\mathrm{AC}$ current of $1 \mathrm{~A}$ in a magnetic flux density of $6.35 \mathrm{kGs}$.

\section{Acknowledgment}

This work was supported in part by the National Natural Science Foundation of China (NSFC) (Grant Nos. 61875246 and 61675091) and the Science and Technology Plan of Guangzhou (Grant No. 201707010338).

Open Access This article is distributed under the terms of the Creative Commons Attribution 4.0 International License (http://creativecommons.org/licenses/by/4.0/), which permits unrestricted use, distribution, and reproduction in any medium, provided you give appropriate credit to the original author(s) and the source, provide a link to the Creative Commons license, and indicate if changes were made.

\section{References}

[1] X. Li and H. Ding, "All-fiber magnetic-field sensor based on microfiber knot resonator and magnetic fluid," Optics Letters, 2012, 37(24): 5187-5189.

[2] L. Sun, S. Jiang, and J. R. Marciante, "All-fiber optical magnetic-field sensor based on Faraday rotation in highly terbium-doped fiber," Optics Express, 2010, 18(6): 5407-5412.

[3] B. O. Guan and S. N. Wang, "Fiber grating laser current sensor based on magnetic force," IEEE Photonic Technology Letters, 2010, 22(4): 230-232.

[4] M. Yang, J. Dai, C. Zhou, and D. Jiang, "Optical fiber magnetic field sensors with TbDyFe magnetostrictive thin films as sensing materials," Optics Express, 2009, 17(23): 20777-20782.

[5] W. He, L. Cheng, Q. Yuan, Y. Liang, L. Jin, and B. O. Guan, "Magnetostrictive composite material-based polarimetric heterodyning fiber-grating laser miniature magnetic field sensor," Chinese Optics Letters, 2015, 13(5): 050602.

[6] M. N. Deeter, A. H. Rose, and G. W. Day, "Fast, sensitive magnetic-field sensors based on the Faraday effect in YIG," Journal of Lightwave Technology, 1990, 8(12): 1838-1842.

[7] J. E. Lenz, "A review of magnetic sensors," Proceedings of the IEEE, 1990, 78(6): 973-989.

[8] J. Noda, T. Hosaka, Y. Sasaki, and R. Ulrich, "Dispersion of Verdet constant in stress-birefringent silica fibre," Electronics Letters, 1984, 20(22): 906-908.

[9] T. Yoshino, T. Hashimoto, M. Nara, and K. Kurosawa, "Common path heterodyne optical fiber sensors," Journal of Lightwave Technology, 1992, 10(4): 503-513.

[10] S. Qiu, Q. Liu, F. Xu, and Y. Lu, "Ampere force based photonic crystal fiber magnetic field sensor," Sensors and Actuators A: Physical, 2014, 210(1): 95-98.

[11] Z. Liu, H. Cao, and F. Xu, "Fiber-optic Lorentz force magnetometer based on a gold-graphene composite membrane," Applied Physics Letters, 2018, 112(20): 203504.1-203504.5.

[12] K. D. Oh, J. Ranade, V. Arya, A. Wang, and R. O. Claus, "Optical fiber Fabry-Perot interferometric sensor for magnetic field measurement," IEEE Photonics Technology Letters, 1997, 9(6): 797-799.

[13] B. O. Guan, L. Jin, Y. Zhang, and H. Y. Tam, "Polarimetric heterodyning fiber grating laser sensors," Journal of Lightwave Technology, 2011, 30(8): 1097-1112. 
[14] Y. Zhang, B. O. Guan, and H. Y. Tam, "Ultra-short distributed Bragg reflector fiber laser for sensing applications," Optics Express, 2009, 17(12): 10050-10055.

[15] T. Zhang, J. Zhang, L. Cheng, Y. Liang, L. Jin, H. Liang, et al., "Response of an erbium-doped dual-polarization fiber laser to a perpendicular gradient magnetic field," Optics Letters, 2017, 42(22): 4675-4678.

[16] M. Poggio and C. L. Degen, "Force-detected nuclear magnetic resonance: recent advances and future challenges," Nanotechnology, 2010, 21(34): 342001.

[17] C. L. Degen, M. Poggio, H. J. Mamin, C. T. Rettner, and D. Rugar, "Nanoscale magnetic resonance imaging," Proceedings of the National Academy of Sciences, 2009, 106(5): 1313-1317.
[18] P. Zhang, M. Tang, F. Gao, B. Zhu, S. Fu, J. Yang, et al., "An ultra-sensitive magnetic field sensor based on extrinsic fiber-optic Fabry-Perot interferometer and Terfenol-D," Journal of Lightwave Technology, 2015, 33(15): 3332-3337.

[19] B. Zhang, L. Cheng, Y. Liang, L. Jin, T. Guo, and B. O. Guan, "Low-frequency vibration measurement by a dual-frequency DBR fiber laser," Photonic Sensors, 2017, 7(3): 206-210.

[20] B. O. Guan, Y. N. Tan, and H. Y. Tam, "Dual polarization fiber grating laser hydrophone," Optics Express, 2009, 17(22): 19544-19550.

[21] L. Cheng, Z. Guo, J. Han, L. Jin, and B. O. Guan, "Ampere force based magnetic field sensor using dual-polarization fiber laser," Optics Express, 2013, 21(11): 13419-13424. 